Int. J. Dev. Biol. 56: 959-967 (2012)

doi: $10.1387 / \mathrm{ijdb} .120134 \mathrm{jd}$

\title{
Involvement of adipokines, AMPK, PI3K and the PPAR signaling pathways in ovarian follicle development and cancer
}

\author{
JOËLLE DUPONT*, ${ }^{*}$, MAXIME REVERCHON ${ }^{1}$, LUCIE CLOIX ${ }^{1,2}$, PASCAL FROMENT ${ }^{1}$ and CHRISTELLE RAMÉ ${ }^{1}$ \\ ${ }^{1}$ Unité de Physiologie de la Reproduction et des Comportements, \\ Institut National de la Recherche Agronomique, UMR85, Nouzilly, France and \\ ${ }^{2}$ Service de médecine interne, diabétologie, endocrinologie CHRU Bretonneau, Tours, France
}

\begin{abstract}
The physiological mechanisms that control energy balance are reciprocally linked to those that control reproduction, and together, these mechanisms optimize reproductive success under fluctuating metabolic conditions. Adipose tissue plays an important role in this regulation. Indeed, it releases a variety of factors, termed adipokines that regulate energy metabolism, but also reproductive functions. This article summarizes the function and regulation of some bettercharacterized adipokines (leptin, adiponectin, resistin, visfatin, chemerin and apelin) involved in ovarian follicle development. The follicle appears to use various "nutrient sensing" mechanisms that may form the link between nutrient status and folliculogenesis. This review examines evidence for the presence of pathways that may sense nutrient flux from within the follicle including the PI3K/ Akt pathway, adenosine monophosphate-activated kinase (AMPK), and peroxisome proliferatoractivated receptors (PPARs). It also reviews current information on the role of these adipokines and signalling pathways in ovarian cancers.
\end{abstract}

KEY WORDS: metabolic sensor, ovarian tumorigenesis, follicle growth, granulosa cell, theca cell, oocyte

\section{Introduction}

Reproductive functions are dependent on energy resources. The role of weight, body composition, fat distribution and the effect of diet have been largely investigated in women and domestic female animals where their alterations may induce abnormalities in timing of sexual maturation and fertility. However, the cellular mechanisms involved in the fine coordination of energy balance and reproduction are largely unknown. It is clear that the brain and hypothalamic structures receive endocrine and/or metabolic signals providing information on the nutritional status and the degree of fat stores. However, it is now widely accepted that there are direct nutritional effects on ovarian development and follicular atresia, which regulate production of germ cells. Thus, it suggests that there are specific energy sensing mechanisms in the follicle. Several candidates have been involved as possible link between nutritional state and the functions of the different ovarian cells. Among these energy sensors, there are factors released by the adipose tissue called adipokines (leptin, adiponectin, resistin, visfatin, chemerin...) but also various signalling pathways including AMPK, PPARs, PI3K/
Akt/PTEN/Foxo that sense nutrient status in the follicle.

This paper reviews earlier and contemporary studies on the effects of these energy sensors on the growth and development of ovarian follicles and more precisely we focused on the functions of granulosa and theca cells and oocyte in different species. In the ovary, bidirectional communications between germ cells (oocytes) and somatic cells (granulosa cells) are crucial to oocyte maturation,

Abbreviations used in this paper: AMPK, adenosine monophosphate activated-kinase; APJ, apelin receptor; CMKLR, chemokine-like receptor; EOC, epithelial ovarian cancer; FAS, fatty acid synthase; Foxo, forkhead box, sub-group O; FSH, folliclestimulating hormone; GSK, glycogen synthase kinase; GVBD, germinal vesicle breack down; IGF, insulin-like growth factor; LH, luteinizing hormone; LPA, lyposaccharide; MAPK ERK, mitogen-activated protein kinase extracellular signal-regulated kinases; mTor, mammalian target of rapamycin; Ob-R, obese gene receptor; PDK, 3-phosphoinositide-dependent kinase; PI3K, phosphatidylinositol 3-kinase; PIP2, phosphatidylinositol 4,5-biphosphate; PPAR, peroxisome proliferator-activated receptor; PTEN, phosphatase and tensin homolog Ob-R, obese gene-receptor; RARRES, retinoic acid receptor responder; TIG, tazaroteneinduced gene; TZD, thiazolidinediones; TSC, tuberous sclerosis; STAT, signal transducer and activator of transcription.

*Address correspondence to: Joëlle Dupont. Unité de Physiologie de la Reproduction et des Comportements, Institut National de la Recherche Agronomique, 37380 Nouzilly, France. Tel: 33-2-4742-7789. Fax: 33-2-4742-7743. e-mail: jdupont@ tours.inra.fr

Final, author-corrected PDF published online: 5 February 2013.

ISSN: Online 1696-3547, Print 0214-6282 
follicle growth and ovulation (Binelli and Murphy, 2010). Oocytes are derived from primodial germ cells, oogonia, which expand through mitosis in the fetal ovary. The germ cells become oocytes once they enter meiosis at birth. They become surrounded by a layer of epithelial cells (granulosa) and are now called primordial follicles. At puberty, primordial follicles resume growth and acquire many layers of granulosa cells to reach the ovulatory stage at each cycle. Ovarian follicular growth and ovulation are dependent on the growth and proliferation of granulosa cells. The period of follicular growth is characterized by mitotic activity of granulosa cells and the transformation of surrounding stroma into layers of thecal cells.

Some of these mediators of energy balance have also been described in the proliferation, migration and invasion of cancer cells. Epidemiological studies have suggested that obesity is associated with ovarian cancer. Both benign and malignant ovarian tumors can arise from each of the three ovarian cell types (germ cells, endocrine and interstitial cells, and epithelial cells (Romero and Bast, 2012). Germ cell tumors account for 3-5\% of ovarian cancers, endocrine and interstitial cell tumors represent approximately $7 \%$ of all ovarian malignancies and epithelial ovarian cancers include approximately $90 \%$ of malignant ovarian tumors. Despite rapid advances in understanding ovarian cancer etiology, epithelial ovarian cancer (EOC) remains the most lethal form of gynecologic cancers. Epithelial ovarian cancer (EOC) is the fourth leading cause of cancer-related death in women in the USA and the leading cause of gynecologic cancer death. Ovarian cancer is the sixth most common cancer and the fifth leading cause of cancer-related deaths among women in developed countries. Malignant transformation of normal ovarian epithelial cells is caused by genetic alteration that disrupts proliferation, programmed cell death and senescence. Two groups of epithelial ovarian cancers have been distinguished: type I low-grade cancers that present in early stage, grow slowly, and resist conventional chemotherapy but may respond to hormonal manipulation; and type II high-grade cancers that are generally diagnosed in advanced stage and grow aggressively but respond to chemotherapy. There has been considerable interest in the potential role of the adipokines but also in their signaling pathways such as AMPK, PPARs, and PI3K/Akt in the development of anti-cancers drugs.

\section{Role of adipokines in the development of the ovarian follicle}

The body mass index and especially the lean/fat ratio are determinants of female fertility. Adipose tissue is now hypothesized to be the largest endocrine organ in the body, secreting a large number of biologically important molecules termed adipokines. Adipose tissue acts as a store of energy and as an active endocrine organ. Adipokines modulate lipid and glucose metabolism and insulin sensitivity. In addition to their well established role in controlling adipose tissue physiology, adipokines have been shown to be involved in regulation of the reproductive functions. During the last years, roles of new adipokines have been emerging in the field of fertility and reproduction and more precisely on the ovarian functions (Campos et al., 2008).

\section{Leptin}

In 1994, leptin is the first adipokine claimed to be the "missing link" between fat and reproduction. Leptin is a $16 \mathrm{kDa}$ peptide hormone secreted mainly from adipose tissue which plays an integral role in the regulation of body weight and energy expenditure. Some evidence suggests that leptin is also an important signal in female reproduction, including control of ovarian function (Brann et al., 2002). The leptin receptor (Ob-R), product of the diabetes $(\mathrm{db})$ gene, is a member of the class I cytokine receptor superfamily, with six known isoforms. Ob-R expression has been observed in many tissues including the hypothalamus and peripheral tissues including the ovary (Brann et al., 2002). Of the known isoforms, only the full-length form $(\mathrm{Ob}-\mathrm{Rb})$ contains the intracellular domains necessary to mediate signal transduction through both the signal transducer and activator of transcription 3 (STAT3), and the MAP kinase pathway.

A significant body of work has shown that $O b-\mathrm{R}$ is expressed in the ovary. Leptin receptor transcripts are present in both human and rat granulosa and theca cells, as well as in cattle and porcine granulosa and theca cells. Ob-Ra and $\mathrm{Rb}$ levels in the ovary vary during the estrous cycle in rodent and cattle. Among the hormonal signals informing the reproductive axis about nutritional status, leptin is growing emerging as a convincing hypothesis. Plasma levels of leptin are correlated with the degree of obesity and are regulated by feeding and fasting. In the leptin deficient female ob/ob mice, treatment with leptin, increases serum levels of LH and ovarian and uterine weight compared to par-fed controls, and restores fertility. A direct ovarian action of leptin has also been demonstrated in rat ovarian granulosa cells, where leptin counteracts the synergistic effect of IGF-1 on FSH-stimulated estradiol and progesterone production. In human, leptin reduces the production of estradiol by granulosa cultured cells (Agarwal et al., 1999). In bovine theca cells, leptin reduces androgen production induced by $\mathrm{LH}$. The presence of Ob-R mRNA (Craig et al., 2004) and protein has been detected in cattle, mouse, rat, and human oocytes, suggesting that the oocyte may be capable of responding to leptin. In porcine oocytes, Ob-R expression is dependent on the stage of follicular development and oocyte maturation. Leptin protein has been detected in the oocyte by immunofluoresence, however others have been unable to detect its mRNA transcript in the oocyte by nested RT-PCR, suggesting it may be produced elsewhere and transported into the oocyte. In the human ovary, leptin is produced by ovarian somatic cells and is present in follicular fluid at concentrations similar to serum, making leptin spatially available for the oocyte. In mice, leptin has been shown to increase the rate of meiotic resumption in preovulatory follicle-enclosed oocytes, presumably via indirect actions on the theca cells (Ryan et al., 2002). In pig, it facilitates oocyte maturation through MAP kinase pathway (Craig et al., 2004).

\section{Adiponectin}

Adiponectin is a novel adipocytokine that was identified in 1995 (Scherer et al., 1995) and 1996. It is the most abundant protein secreted by adipose tissue (accounting for approximately $0.01 \%$ of total plasma protein at around $5-10 \mu \mathrm{g} / \mathrm{mL}$ in human). Its circulating concentration is inversely related to adiposity and body mass index. It plays an important role in regulating energy homeostasis, specifically lipid and glucose metabolism. Adiponectin activates AMP-activated protein kinase (AMPK) and peroxisome proliferator-activated receptors alpha (PPAR $\alpha$ ). Two adiponectin receptors (AdipoR1 and AdipoR2) have been identified and found to have functional differences. The predicted structure of the two receptors is similar to the topology of G-protein coupled receptors, 
but the intracellular/extracellular orientation of $\mathrm{N}$-terminus and $\mathrm{C}$ terminus is the opposite. The expression of all or some genes of the adiponectin system was identified in the ovary of various species including rat, pig, chicken, human and recently cattle (Maillard et al., 2010, Tabandeh et al., 2010). Except in bovine species, adiponectin expression is absent/low in chicken, mouse and human granulosa cells and cumulus cells. The expression of adiponectin and its receptors changes in different cells of bovine ovary during morphological and physiological development (Tabandeh et al., 2010). Furthermore, adiponectin receptors have been shown to be expressed by oocytes and early embryos in porcine and mice species (Chappaz et al., 2008). mRNA as well as protein for both adiponectin receptors are expressed in mouse ovulated oocytes and in in vivo derived preimplantation embryos at all examined stages of development. A few studies have demonstrated the relationship between adiponectin and female reproduction. Recently, some evidence suggests that adiponectin could be involved in the control of reproductive functions. Indeed, overexpression of circulating adiponectin impairs female fertility in mice, whereas the absence of adiponectin has no effect. Additionally, in vitro studies showed that adiponectin decreases androgen and progesterone production induced by insulin in bovine theca cells. Furthermore, our team has found in rat, chicken and human, that physiological levels of recombinant human adiponectin ( 5 or $10 \mu \mathrm{g} / \mathrm{ml}$ ) are able to increase progesterone and/or estradiol secretions in response to insulin-like growth factor-I (IGF-I) in cultured granulosa cells. Several reports in different species, including humans, have indicated that adiponectin can modulate not only granulosa cell steroidogenesis but also the expression of genes associated with ovulation. There is also evidence that the functions of the two adiponectin receptors in granulosa cells may differ. In the human ovarian granulosa-like tumor cell-line (KGN), AdipoR1 seems to be involved in the cell survival whereas AdipoR2, through MAPK ERK1/2 activation could regulate steroid production (Pierre et al., 2009). In bovine species, adiponectin decreased insulin-induced steroidogenesis and increased IGF-1-induced proliferation of cultured granulosa cell through a potential involvement of ERK1/2 MAPK pathway, whereas it did not modify oocyte maturation and embryo development in vitro (Maillard et al., 2010). In porcine species, adiponectin stimulates oocyte meiotic maturation and in vitro embryo development (Chappaz et al., 2008). Also, in mice, the addition of adiponectin to mouse cumulus-oocyte-complex improves oocyte quality as assessed by fertilization and early stages of embryo growth. Thus, addition of adiponectin to in vitro maturation media could improve the developmental competence of in vitro matured oocytes in human infertility care. Of clinical relevance, adiponectin levels are reduced in women with polycystic ovarian syndrome compared with fertile women, which is possibly linked with elevated androgens and obesity, and altered adipose tissue functions.

\section{Resistin}

Resistin was discovered in 2001 as a Thiazolidinedionesdownregulated gene in mouse adipocytes (Steppan et al., 2001). Resistin is an adipocyte-derived cytokine that plays an important role in the development of insulin resistance and obesity in rodents. Resistin is a cysteine-rich protein of around $12 \mathrm{kDa}$ that belongs to a family of polypeptides named resistin-like molecules. In mice, adipocytes may be the major source of resistin, whereas in humans, resistin mainly come from monocytes and macrophages. Despite much research on resistin's action, the receptor(s) mediating its biological effects has not yet been identified, and little is known on the intracellular signaling pathways activated by this protein. In cultured human theca cells, $17 \alpha$-hydroxylase activity is increased by resistin in the presence of forskolin or forskolin \pm insulin, suggesting a role of resistin in stimulation of androgen production by theca cells (Munir et al., 2005). Some studies have shown elevated concentrations of serum resistin in women with polycystic ovary syndrome, which is known to be associated with insulin resistance, hyperinsulinemia and hyperandrogenism. In cow and rat, resistin mRNA and protein are present in various ovarian structures such as theca cells and oocyte. However, resistin expression is speciesdependent in granulosa cells (Maillard et al., 2011). In both species, recombinant resistin can modulate steroidogenesis and proliferation in basal state or in response to IGF1 in vitro (Maillard et al., 2011). In bovine species, resistin inhibits in vitro steroidogenesis of undifferentiated (small follicles) granulosa cells and inhibits mitogenesis of differentiated (large follicle) granulosa cells suggesting that the ovarian response to resistin is altered during follicular development (Spicer et al., 2011). However, in this latter species, it had no effect on IGF-I- or insulin-induced progesterone and androstenedione production by theca cells (Spicer et al., 2011). The role of resistin in oocyte is still unknown.

\section{Visfatin}

Visfatin (52kDa), discovered in 2005, also known as nicotinamide phosphoribosyltranferase (nampt) or pre B cell colony enhancing factor (PBEF) is a multifunctional protein predominantly expressed in the visceral adipose tissue (Dahl et al., 2012). Visfatin concentration in follicular fluid is positively correlated with the number of follicles retrieved from women undergoing in vitro fertilization, suggesting that visfatin may act in ovarian function, like follicles growth up, cells differentiation, oocyte maturation or ovulation. A recent study showed that visfatin mRNA is expressed in human granulosa cells and a hCG treatment for $24 \mathrm{~h}$ increased considerably visfatin mRNA expression suggesting a role of visfatin in final follicle maturation or ovulation (Shen et al., 2010). In our laboratory, we have showed that visfatin increases IGF-1-induced steroid production in primary human granulosa cells and in the human granulosa KGN cell-line.

\section{Chemerin}

Chemerin also known as RARRES2 or TIG2, was discovered in 1997. It is a secreted 18-kDa inactive pro-protein and cleaved into a $16-\mathrm{kDa}$ active form by extracellular serine protease cleavage, which is present in plasma and serum (Goralski et al., 2007). The plasma chemerin levels are significantly higher in obese subjects than those with normal weight. It was also recently reported that circulating chemerin levels increased in PCOS non obese patients versus control. Chemerin and its receptor, CMKLR1 are present in human ovarian follicles (mainly in granulosa and theca cells) and in KGN cells. In primary human granulosa cells, chemerin decreases IGF-1-induced thymidine incorporation, progesterone and oestradiol production through a decrease in the phosphorylation of IGF-1R beta subunit and MAPK ERK1/2 signaling pathways (Reverchon et al., 2012).

\section{Apelin}

In 1998, Tatemoto et al., (1998) isolated a novel endogenous apelin peptide from the bovine stomach, which was found to be 
a ligand of the apelin receptor (also known as the APJ receptor) (Tatemoto et al., 1998). APJ receptor is an orphan G proteincoupled receptor. Apelin is produced through processing from the C-terminal portion in the pre-proprotein consisting of 77 amino acid residues and exists in multiple molecular forms. APJ receptor most closely resembles the angiotensin type 1 receptor; however, the APJ receptor does not bind to angiotensin II. Apelin peptide is a potent angiogenic factor inducing endothelial cell (EC) proliferation, EC migration, and the development of blood vessels in vivo. The apelin levels produced by adipocytes, vascular stromal cells, and the heart, are increased with high insulin levels, obesity or insulinresistance. The APJ receptor is expressed in granulosa cells, and both apelin and the APJ receptor are expressed in the theca cell layer of follicles in bovine ovaries (Shimizu et al., 2009). In theca cells, apelin and APJ expression is induced by LH. In granulosa cells, the increase in expression of APJ receptor mRNA is associated with the follicular atresia (Shimizu et al., 2009). The expression of apelin and APJ in mature follicles suggests that the apelin/APJ system could play an important role during follicle selection and dominance in the cow (Schilffarth et al., 2009).

Thus, various in vitro studies have shown that adipokines are able to affect directly the ovarian functions. Some of these effects are summarized in the Fig. 1.

\section{Role of adipokines in ovarian cancer}

There is some evidence that adipokines are involved in the development and the progression of several cancers including breast, prostate and colon. More specifically, in patients with these cancers, the plasma adiponectin levels are significantly reduced. The roles of adipokines in ovarian cancers have been much less studied. Leptin is suggested to be associated with cancer development and progression in many epithelial cancers including epithelial ovarian cancer (EOC) (Mor et al., 2005). In vitro studies consistently show that leptin has angiogenic and proliferative potential in cancer (Choi et al., 2005). There is some evidence that plasma adiponectin concentrations are significantly lower in serum of patients with early-stage ovarian cancer compared with healthy women. Adiponectin has been shown to exhibit anti-tumorigenic properties, such as suppression of tumor growth, cellular proliferation in addition to inducing cell growth arrest and apoptosis. In leghorn chicken ovarian tumors, adipoR1 mRNA expression is significantly decreased as compared to non-tumorous chicken ovaries (Ocon-Grove et al., 2008). The authors of this latter study speculate that adiponectin signaling in the ovary is likely to limit ovarian tumor growth, possibly by suppressing tumor cell proliferation and inducing apoptosis. Visfatin protein expression is significantly increased in ovarian serous adenocarcinomas as compared to benign ovarian tissue (Shackelford et al., 2010). So far, no data (plasma or change of expression) about chemerin, resistin and apelin and ovarian cancers are known.

Most of these adipokines (leptin, adiponectin, resistin) mediate their effect through the same signaling pathways named AMPK, PI3K, MAPK and PPAR that are also involved in the ovarian follicle development and cancer.

\section{Role of adenosine monophosphate-activated protein kinase (AMPK) in ovarian follicle development}

AMPK is a serine/threonine kinase and is highly conserved throughout eukaryotes. It is a heterotrimeric protein consisting of a catalytic alpha - and regulatory beta - and gamma -subunits. It is known as the fuel gauge of the cell because it mediates a nutrient signaling pathway that senses cellular energy status. AMPK is an important cellular energy sensor that is activated in response to deficits in ATP and acts by shutting down energy consumption and turning on energy-generating pathways (Hardie, 2003). It is activated during physiopathological situations (food restriction, exercise, stress), by hormones (leptin, adiponectin..) or by pharmacological agents (5-aminoimidazole-4-carboxamide-1- $\beta$-D-riboside [AICAR], metformin, thiazolidinediones). The potential involvement of AMPK in reproduction and more specifically in ovarian function is recent.

In various animal species (human, rodents, cow and chicken), AMPK has been identified in different cell types of the follicle (oocyte, cumulus cells, granulosa and theca) and in the corpus luteum (Tosca et al., 2005). In human, AMPK activation is decreased in PCOS thecal cells, and metformin treatment of these cells increases AMPK activity. Thus, activation of AMPK in the ovary itself could be a reason why patients with PCOS who are treated with metformin experience amelioration of ovulatory dysfunction.

AMPK has been extensively studied in vitro steroidogenesis of granulosa cells of rat, chicken and bovine species and oocyte maturation in the mouse, cow and pig (Downs et al., 2002, Mayes et al., 2007, Tosca et al., 2007a). In rat and cattle, AMPK inhibits the secretion of progesterone and / or that of oestradiol in response to the AICAR or metformin (Tosca et al., 2007a, Tosca et al., 2005). This decrease is partly explained by an inhibition of the expression

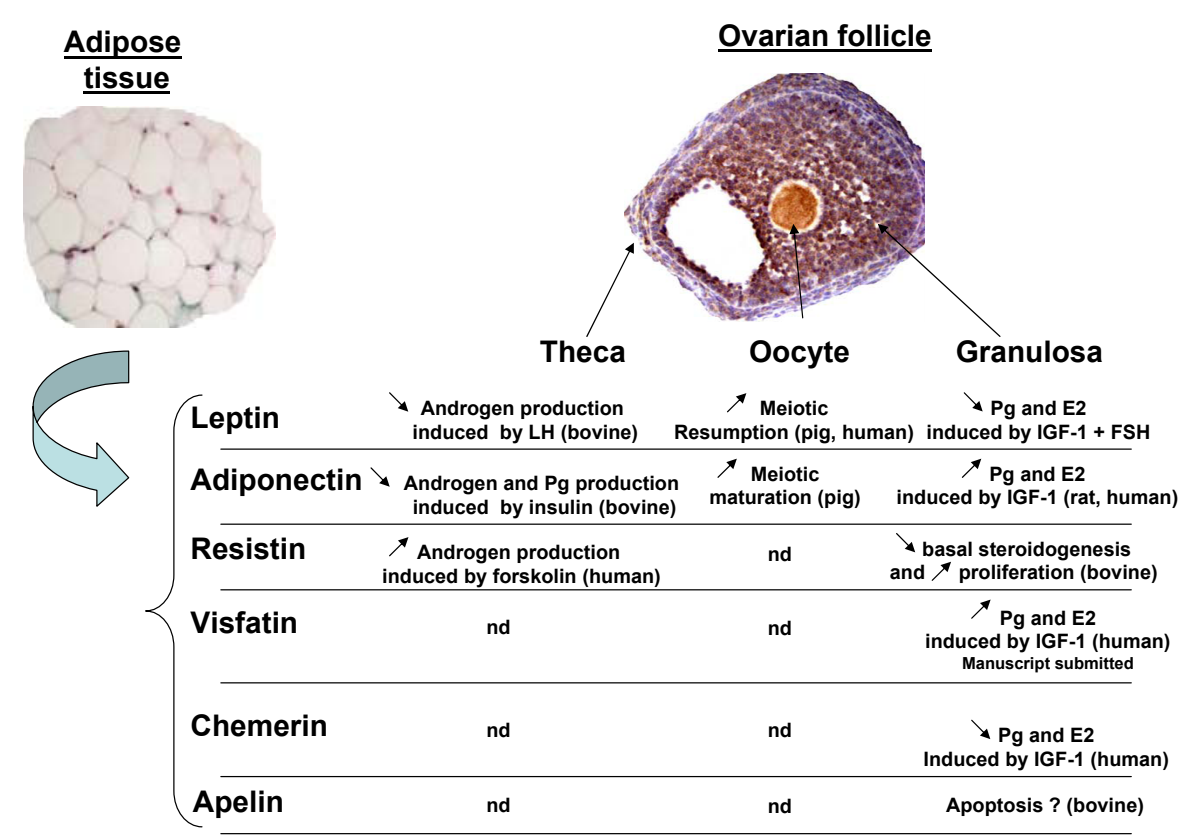

Fig. 1. In vitro effects of some adipokines on the functions of granulosa and theca cells and oocytes in different species. ND, Not yet determined. 
A

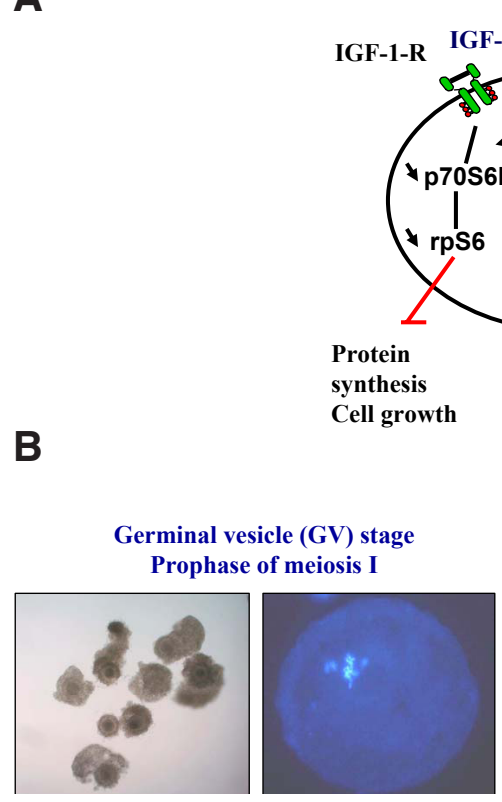
Metformin

Fig. 2. In vitro effects of metformin induced-AMPK activation in bovine granulosa cells and oocyte. (A) Metformin induced AMPK activation reduces IGF-1 and FSH-induced estradiol and progesterone secretion through inhibition of MAPK ERK1/2 phosphorylation (Tosca et al., 2007). It also reduces IGF-1-induced protein synthesis and cell growth through an inhibition of p70S6K and ribosomal S6 protein phosphorylation (Tosca et al., 2010). (B) AMPK activation in response to metformin blocks meiotic progression at the GV stage (adapted from Tosca et al., 2007). was also found in the corpus luteum in rat. The corpus luteum is characterized by steroid production. AMPK could control at this level the process of luteinization, for example by modulating some components of the cholesterol production pathways. AMPK and PPARs (peroxisome proliferatoractivated receptors) are tightly linked, some drugs activating AMPK activate also PPARgamma and the activation of PPAR in cow increases the production of progesterone by the corpus luteum. Thus, AMPK could control steroidogenesis in ovarian cells (granulosa, theca cells and corpus luteum cells) and germ cell maturation.

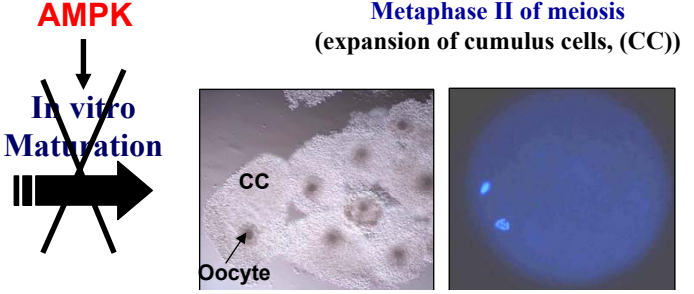

of some factors of steroidogenesis (3 $\beta$ HSD [3 $\beta$-hydroxysteroid dehydrogenase-], P450scc [p450 side chain cleavage] or StAR [Steroidogenic acute regulatory protein]) and by inhibition of MAPK / ERK (mitogen-activated protein kinase / extracellular-regulated kinase). A schema is represented in Fig. 2A to summarize the effects of metformin-induced AMPK activation in bovine granulosa cell steroidogenesis. In chickens, the activation of AMPK decreases in granulosa cells during folliculogenesis terminal, suggesting a role of the kinase in the pre-ovulatory period (period of the rapid growth of the follicle) in this species. AlphaAMPK is also involved in the regulation of ovarian cell proliferation and apoptosis. In cultured bovine granulosa cells, metformin inhibits proliferation and protein synthesis in response to IGF-1 through an AMPK-dependent mechanism as indicated in the Fig. 2A (Tosca et al., 2010). Furthermore, in primary rat granulosa cells, FSH promotes granulosa cell proliferation by increasing cyclin D2 mRNA expression and by reducing p27 kip expression by inhibiting AMPK activation through an Akt-dependent pathway (Kayampilly and Menon, 2009).

Some studies have also established a link between AMPK and meiotic maturation of the oocyte. In mice, AMPK activation enhances the resumption of meiosis (acceleration of rupture of the nuclear membrane or GVBD stage (germinal vesicle breack down), whereas in pigs or cattle, a pharmacological activation of the AMPK blocks the nuclear maturation of the oocyte (blocked in prophase of first meiotic division stage or germinal vesicle (GV) as indicated in Fig. 2B (Bilodeau-Goeseels 2011, Mayes et al., 2007, Tosca et al., 2007b). Although there are differences between species, AMPK appears to be important in the transition GV-GVBD during oocyte nuclear maturation. The role of AMPK in nuclear maturation could therefore condition early embryonic development. Finally, AMPK complex), involved in the protein synthesis by stress or pharmacological activators in via regulation of $\mathrm{mTOR} / \mathrm{S} 6 \mathrm{~K}$ and translation elongation factor 2 Second, AMPK inhibits expression and/or activity of enzymes, Fatty Acid Synthase and mTOR that are increased in cancers. Third, in vitro AMPK activation inhibits the growth of some cancer cells. Recent studies introduced AMPK as an important regulatory factor in cell migration through the transcriptional up-regulation of integrins (Kim et al., 2011). It could be one possible mechanism by which metformin exerts its potential anti-neoplastic properties. In ovarian cancer cells, AMPK is an important regulatory factor for cell migration through $\mathrm{LPA}_{2}$-mediated cytoskeleton reorganization and tumor metastasis in vivo. Thus, AMPK may be a key therapeutic target for the control of ovarian cancer progression (Kim et al., 2011).

\section{Role of PI3K signaling in the ovarian follicle and cancer}

The phosphoinositide-3 kinase (PI3K) pathway plays an important role in many biological functions including metabolic control, immunity, and cancer (Katso et al., 2001). Although the basic framework of PI3K signaling is well understood, much remains to be learned about its role in reproductive biology. PI3K is activated by IGF-1 and FSH, which normally regulates granulosa cell differentiation and follicular development after antrum formation but also by $\mathrm{LH}$, which is essential for ovulation.

Mammalian phosphatidylinositol 3-kinases (PI3Ks) are a family of lipid kinases able to phosphorylate the 3 position hydroxyl group of the inositol ring of phosphatidylinositol (Ptdlns). The phosphoinositol-3-kinase family is divided into three different classes: Class I, Class II, and Class III, based on their substrate 
preferences, primary structure and regulation. The class I PI3K is the best studied to regulate the ovarian follicle. There are two subgroups of class I PI3Ks, class IA and class IB. Class IA PI3Ks are activated by growth factors receptors including insulin and IGF-1 receptors that play a key role in the follicle development. Class IB PI3Ks are activated by G-protein coupled receptors (GPCRs) including $\mathrm{FSH}$ and $\mathrm{LH}$ receptors that control differentiation and secretion of ovarian follicular cells.

Once activated, PI3K phosphorylates the membrane phospholipid, phosphatidylinositol4,5-biphosphate (PIP2), thereby producing phosphatidylinositol3,4,5-triphosphate (PIP3), which in turn acts as a second messenger to activate PDK1 (3-phosphoinositide-dependent kinase-1). PDK1 phosphorylates many important kinases such as Akt/PKB and p70 S6 kinase in their respective activation loops. Activated Akt directly phosphorylates various substrates including GSK3 (glycogen synthase kinase-3), Bad, the proapoptotic forkhead transcription factors (FOXO1, FOXO3a and FOXO4) and the tuberous sclerosis 2(TSC2). The inactivation of GSK3 leads to the dephosphorylation and activation of glycogen synthase and hence to an acceleration of glycogen synthesis. The phosphorylation of FOXOs by Akt in response to growth factors leads to the exclusion of FOXOs from the nucleus and consequently prevents their actions. Akt also exerts anti-apoptotic effects by phosphorylating Bad. In parallel, Akt activates the mTORC complex (mTOR, mammalian target of rapamycin and its regulatory proteins associated) by phosphorylation of TSC2 and destabilization of the TSC1/TSC2 complex. mTORC may then increase phosphorylation of the initiation factor 4E-binding protein (4E-BP1) and S6K (S6 kinase) promoting protein synthesis, ribosome biogenesis and autophagy. The biological effects of the PI3K pathway can be negatively regulated at the level of PIP3 by phospholipid phosphatases, including the phosphatase and tensin homolog (PTEN) and SH2-containing 5'-phophatase-2 (SHIP2), which dephosphorylate and inactivate PIP3, as indicated in Fig. 3.

\section{PI3K signaling and follicle growth}

Several roles of the PI3K pathway in the ovarian follicle have been recently elucidated by using conditional knockout mouse models as described in the recent review written by Zhen et al., 2012 (Zheng et al., 2012). PTEN is the most studied in the mammalian ovary. The phosphatidylinositol 3-kinase (PI3K) pathway, known to be essential for the regulation of cell proliferation, survival, migration, and metabolism in different tissues, is also likely to play a role in the regulation of the activation of primordial follicles in the mice ovary. Indeed, deletion of Pten (leading to activation of $\mathrm{PI} 3 \mathrm{~K}$ ) specifically in oocytes promptly activates follicular development in mice very early in life (Reddy et al., 2008). This early follicular activation resulting from heightened PI3K-Akt signaling in oocytes lacking PTEN suggests that signaling from oocytes to follicles is a contributing factor in regulating follicular activities. Interestingly, another group found that PI3K-Akt-dependent hyperphosphorylation of the transcription factor Foxo3 also in oocytes induces premature oocyte activation suggesting that Foxo3 acts downstream of PTEN to negatively regulate follicular activation (Adhikari et al., 2009). This irreversible premature oocyte activation leads to rapid depletion of follicle reserve, leading to a demise of ovarian function similar to premature ovarian failure in humans. Although these studies highlight roles of oocyte-specific PTEN in regulating follicular function, another recent study shows that granulosa cell-specific deletion of Pten leads to repressed structural luteolysis and enhanced proliferation of granulosa cells resulting to increased follicle growth, ovulation rate and litter size and prolonged corpora luteal lifespan. It has been shown that the PI3K/PDK1, PI3K/AKT/S6K1, PI3/rpS6 cascade in primary oocytes controls ovarian aging by regulating the survival of primordial follicles. Furthermore, deletion of Tsc1 or Tsc2 specifically from oocytes leads to a global activation of all primordial follicles around the time of puberty, ending up with follicular depletion in early adulthood and causing premature ovarian failure (POF) in mice (Adhikari et al., 2009, Adhikari et al., 2010). Furthermore, Tanaka et al., 2012 have recently showed that conditional deletion of TSC1 by a knock-in allele of the anti-Müllerian hormone type 2 receptor (Amhr2) driving Cre expression and subsequent activation of mTOR in granulosa cells and in oviductal and uterine stromal cells affects fertility in female mice. Heterodimeric complex of tuberous sclerosis complex 1 (TSC1 or hamartin) and TSC2 (or tuberin) is one of the most important sensors involved in the regulation of $\mathrm{mTORC} 1$ activity. Mammalian target of rapamycin (mTOR) is a serine/threonine kinase that regulates cell growth and proliferation in response to diverse signals. mTOR is part of two distinct multiprotein complexes: rapamycin sensitive mTOR complex 1 (mTORC1) and rapamycin insensitive mTORC2. The effects of rapamycin treatment of mice in vivo are consistent with 
effects on follicle growth to ovulatory stages. mTOR inhibition appears to slow the growth of the follicle in two ways: increased cell cycle arrest in the G1 stage of the cell cycle (as previously seen in several different cell types), and via the induction of mitotic anomalies in granulosa cells (Yaba et al., 2008). mTOR is also involved in the meiotic maturation of the oocyte. Indeed, rapamycin inhibits spindle migration and asymmetric division during mouse oocyte maturation via mTOR-mediated small GTPase signaling pathways.

Thus, in the oocyte, the PI3K signaling pathway is essential for the survival (through PDK1, Akt, mTORC1, S6K1 and RPS6) and to maintain the quiescence (through PTEN, Foxo3 and TSC1/TSC2) of primordial follicles as indicated in Fig. 3. In granulosa cells it regulates the proliferation and differentiation of granulosa cells in response to gonadotropins suggesting a role in the cyclic follicle recruitment. In the oocyte, PI3K seems to not be determinant for meiotic resumption.

\section{PI3K signaling and ovarian cancer}

In ovarian cancer, the PI3Kinase/Akt/mTOR activity is frequently elevated, leading to make the cancer more aggressive and facilitating to spread to other organs. Abnormalities of phosphatidylinositol 3 kinase (PI3K) signaling have been detected in both type I and type Il ovarian cancers (for review (Bast and Mills, 2012)). Cells having increased PI-3 kinase activity possess mutated PTEN and have elevated levels of PKB and PDK1 activity, which in turn activates several protein serine/threonine kinases, including PKC, ribosomal S6 kinase, SGK, Rho kinase and PAK1, all of which are associated with increased invasion and metastasis. Moreover, Akt, the most extensively studied downstream target of PDK1, has also been shown to be overexpressed in ovarian carcinomas (Arboleda etal., 2003). The firstindication of aberrations targeting the PI3K pathway in ovarian cancer was the demonstration of AKT2 amplification. In culture of ovarian cancer cells, rapamycin (inhibitor of TORC1) decreased proliferation and activation of TORC1 targets such as

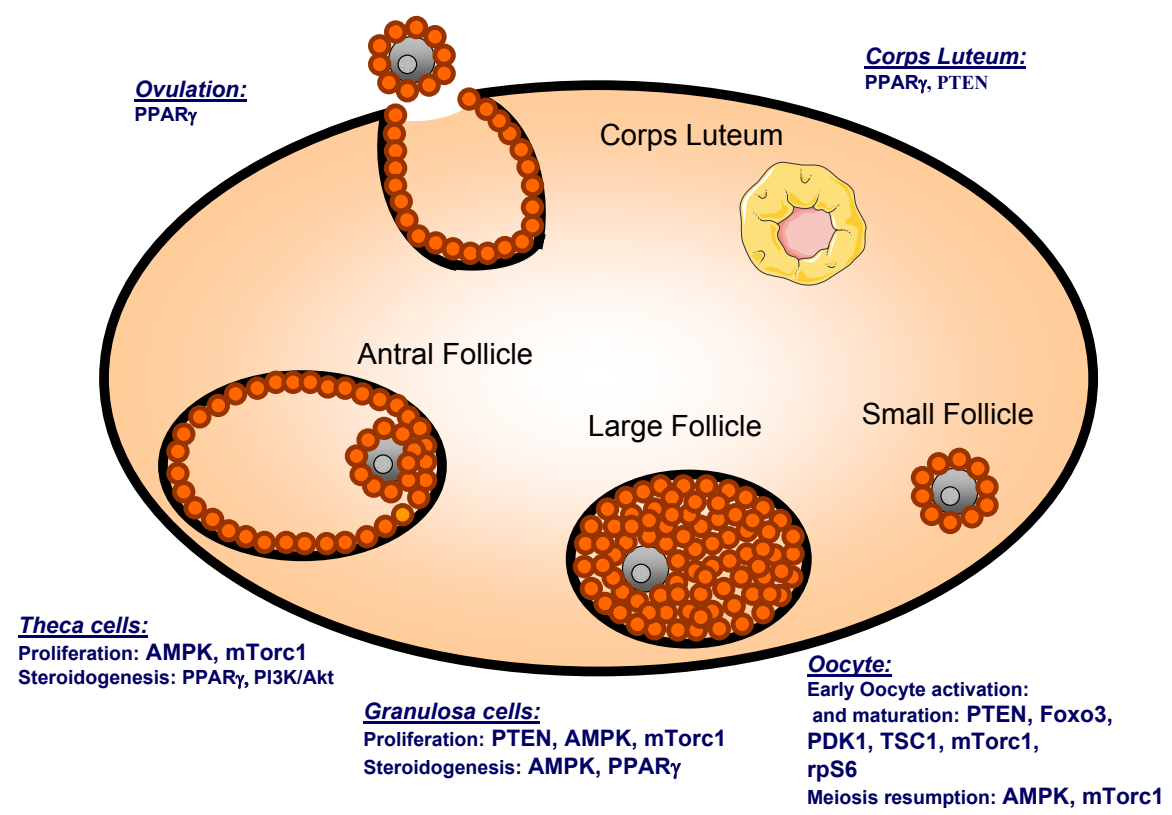

Fig. 4. Some examples of the involvement of AMPK, PI3K/Akt and PPAR $\gamma$ signaling pathways in the different ovarian functions and cell compartments. inhibition of the phosphorylation of S6 (protein involved in protein synthesis). Hence, the use of mTOR inhibitors (rapamycin and its derivatives) is considered to be a good target for ovarian cancer therapy. Neverthess, rapamycin presents some undesirable properties including poor water-solubility, that's why several analogues have been developed such as tensirolimus and everolimus, two synthetic ester analog of rapamycin promising target for anticancer therapy. Theses agents have shown remarkable inhibition of cell proliferation, tumor angiogenesis, and abnormal cell metabolism against ovarian cancers in vitro and in vivo. Indeed, everolimus delayed ovarian tumor development in a transgenic mouse model developing spontaneous epithelial ovarian cancer. Hence, even if all untreated transgenic mice developed advanced ovarian carcinoma, only $30 \%$ of mice administrated with everolimus developed early ovarian carcinoma. Both tensirolimus and everolimus inhibit downstream signalling of mTOR and lead to cell cycle arrest and apoptosis.

Thus, strong associations of deregulated PI3K signaling pathways with ovarian carcinoma have also been well established. To maintain optimal activities of PI3K signaling within the ovary seems to be crucial for maintaining normal development and physiology of the ovary.

\section{Role of peroxisome proliferator-activated receptors in ovarian follicle and cancer}

The peroxisome proliferator-activated receptors (PPARs) are other metabolic sensors that are largely involved in the follicle development. They are transcription factors and members of the nuclear receptor superfamily (Feige et al., 2006). The three closely related PPAR isoforms identified as PPAR $\alpha$, PPAR $\beta$ and PPAR $\gamma$ are encoded by separate genes and fulfill specific functions. As transcription factors requiring activation, the PPARs modulate the expression of target genes in the cytoplasm or in the nucleus in response to ligand binding. Physiological ligands of PPARs include fatty acids and their derivatives, leukotrienes and prostaglandins.

In several species including rat, all three PPAR isotypes are detected in the ovary. PPAR $\gamma$, which has been studied more extensively than the other two isotypes, is detected in the mouse, rat, pig, sheep, cow, bull and human ovary (reviewed in (Komar, 2005)). PPAR $\gamma$ is expressed strongly in the granulosa cells of rat, mouse, and sheep, as well as in oocytes from cattle, zebrafish, Xenopus, and human. PPAR $\gamma$ is detected in different classes of follicles (primary/secondary to preovulatory follicles) and its expression increases with the development of follicles. After the LH surge, PPAR $\gamma$ mRNA expression is downregulated. Results from a study by Cui etal., (2002) indicated that PPAR $\gamma$ plays an important role in normal ovarian function. Indeed, using cre/loxP technology, the expression of PPAR $\gamma$ was disrupted in the ovary, rendering $1 / 3$ of the females sterile, and the remaining females sub-fertile (Cui et al., 2002). Activation of PPAR $\gamma$ by natural and synthetic ligands (rosiglitazone) in the granulosa cells appears to regulate the 
synthesis of steroid hormones (Froment etal., 2003). Thus, PPAR $\gamma$ may be indirectly involved in oocyte maturation via the granulosa cells. PPARs may also limit the synthesis of estradiol by reducing production of androgenic precursors by theca cells due to the expression of PPARs in these cells. Indeed, disruption of PPAR $\gamma$ gene in the ovary using cre/loxP technology led to female subfertility (Cui et al., 2002). On the other hand, PPARs may be directly involved in oocyte maturation and ovulation. Indeed, it has been reported that rosiglitazone, a synthetic PPAR $\gamma$ ligand, at $100 \mu \mathrm{M}$ stimulates AMP-activated protein kinase (AMPK) and enhances the meiotic resumption of mouse oocytes. Furthermore, granulosa cell-specific deletion of PPAR gamma in mice results in marked impairment of ovulation due to defective follicular rupture (Kim et al., 2008). PPAR $\gamma$ is also involved in processes that are critical to normal ovarian function such as angiogenesis, inflammation, and cell cycle control, indicating that PPAR $\gamma$ may be an important player regulating ovarian gene expression.

\section{Peroxisome proliferator-activated receptors and ovarian cancer}

In cancer biology, PPAR $\gamma$ is the most intensively studied PPAR isoform. It is expressed in high levels in different cancer including colon, breast, bladder, prostate, head and neck, cervical and endometrial cancer. PPAR $\gamma$ protein is significantly increased in malignant ovarian tumours (grade 1, 2 and 3) compared to benign tumours and normal ovaries (Zhang et al., 2005). These findings suggest an involvement of PPAR $\gamma$ in the onset and development of ovarian carcinoma. PPAR $\gamma$ heterozygous knockout mice (PPAR $\gamma+/-)$ have an increased susceptibility to develop mammary, ovarian and skin cancer after administration of a carcinogen compared to wild-type mice, suggesting that PPAR- $\gamma$ might also provide a protective effect against ovarian cancer development. PPAR $\gamma$ agonists have been studied for their potential use as cancer therapeutic agents. Ciglitazone and troglitazone cause a decrease in proliferation of three human epithelial ovarian cancer cell lines (Ovcar3, CaOv3, and Skov3), and two human ovarian carcinoma cell lines (ES-2 and PA-1) mainly through cell cycle arrest. In the ovarian carcinoma cells, ligand-mediated PPAR $\gamma$ activation suppress COX-2 expression via the NF $\kappa$ B pathway suggesting that high expression of PPAR $\gamma$ and low expression of COX-2 might play an important role in inhibiting ovarian carcinogenesis. Treatment with CA (clofibric acid), a ligand for PPAR $\alpha$ or pioglitazone, a ligand for PPAR $\gamma$ significantly induces apoptosis in ovarian cancer cells suggesting that these ligands could be promising agents towards a novel therapeutic strategy against ovarian cancer (Shigeto et al., 2007). Despite, PPAR $\beta$ protein is expressed in normal ovaries and ovarian cancer, a higher expression of PPAR $\beta$ may be related with the tumor stage and metastasis of epithelial ovarian carcinoma.

\section{Conclusions}

The adipokines, leptin, adiponectin, resistin, but also visfatin, chemerine and apelin produced by adipose tissue and altered with obesity, clearly influence energy homeostasis but also undoubtedly affect female fertility. More precisely, several in vivo and in vitro studies showed the presence of these adipokines and/or their receptors in the different ovarian cells and suggest that they could directly affect the processes of ovarian steroidogenesis and ovulation. These adipokines mediate their actions by activating different signaling pathways including AMPK, PI3K/Akt and PPARs that are already known to play a crucial role in the ovarian development and follicular atresia, which regulate production of germ cells (Fig. 4). Some of these signaling pathways and adipokines are deregulated in many tumors, including ovarian ones. Thus, they could be considered as attractive targets to be investigated in the development of new therapeutic strategies for the ovarian cancers but also for reproductive pathologies such SOPK.

\section{References}

ADHIKARI, D., FLOHR, G., GORRE, N., SHEN, Y., YANG, H., LUNDIN, E., LAN, Z. GAMBELLO, M.J. and LIU, K. (2009). Disruption of TsC2 in oocytes leads to overactivation of the entire pool of primordial follicles. Mol Hum Reprod 15: 765-770.

ADHIKARI, D., ZHENG, W., SHEN, Y., GORRE, N., HAMALAINEN, T., COONEY, A.J., HUHTANIEMI, I., LAN, Z.J. and LIU, K. (2010). Tsc/mTORC1 signaling in oocytes governs the quiescence and activation of primordial follicles. Hum $\mathrm{Mol}$ Genet 19: 397-410.

AGARWAL, S.K., VOGEL, K., WEITSMAN, S.R. and MAGOFFIN, D.A. (1999). Leptin antagonizes the insulin-like growth factor-I augmentation of steroidogenesis in granulosa and theca cells of the human ovary. $J$ Clin Endocrinol Metab 84: 1072-1076.

ARBOLEDA, M.J., LYONS, J.F., KABBINAVAR, F.F., BRAY, M.R., SNOW, B.E., AYALA, R., DANINO, M., KARLAN, B.Y. and SLAMON, D.J. (2003). Overexpression of AKT2/protein kinase Bbeta leads to up-regulation of beta1 integrins, increased invasion, and metastasis of human breast and ovarian cancer cells. Cancer Res 63: 196-206.

BAST, R.C., JR. and MILLS, G.B. (2012). Dissecting "PI3Kness": The Complexity of Personalized Therapy for Ovarian Cancer. Cancer Discov 2: 16-18.

BILODEAU-GOESEELS, S. (2011). Cows are not mice: the role of cyclic AMP, phosphodiesterases, and adenosine monophosphate-activated protein kinase in the maintenance of meiotic arrest in bovine oocytes. Mol Reprod Dev 78: 734-743.

BINELLI, M. and MURPHY, B.D. (2010). Coordinated regulation of follicle development by germ and somatic cells. Reprod Fertil Dev 22: 1-12.

BRANN, D.W., WADE, M.F., DHANDAPANI, K.M., MAHESH, V.B. and BUCHANAN, C.D. (2002). Leptin and reproduction. Steroids 67: 95-104.

CAMPOS, D.B., PALIN, M.F., BORDIGNON, V. and MURPHY, B.D. (2008). The 'beneficial' adipokines in reproduction and fertility. Int J Obes (Lond) 32: 223-231.

CHAPPAZ, E., ALBORNOZ, M.S., CAMPOS, D., CHE, L., PALIN, M.F., MURPHY, B.D. and BORDIGNON, V. (2008). Adiponectin enhances in vitro development of swine embryos. Domest Anim Endocrinol 35: 198-207.

CHOI, J.H., PARK, S.H., LEUNG, P.C. and CHOI, K.C. (2005). Expression of leptin receptors and potential effects of leptin on the cell growth and activation of mitogen-activated protein kinases in ovarian cancer cells. J Clin Endocrinol Metab 90: 207-210.

CRAIG, J., ZHU, H., DYCE, P.W., PETRIK, J. and LI, J. (2004). Leptin enhances oocyte nuclear and cytoplasmic maturation via the mitogen-activated protein kinase pathway. Endocrinology 145: 5355-5363.

CUI, Y., MIYOSHI, K., CLAUDIO, E., SIEBENLIST, U.K., GONZALEZ, F.J., FLAWS, J., WAGNER, K.U. and HENNIGHAUSEN, L. (2002). Loss of the peroxisome proliferation-activated receptor gamma (PPARgamma) does not affect mammary development and propensity for tumor formation but leads to reduced fertility. $J$ Biol Chem 277: 17830-17835.

DAHL, T.B., HOLM, S., AUKRUST, P. and HALVORSEN, B. (2012). Visfatin/NAMPT: A Multifaceted Molecule with Diverse Roles in Physiology and Pathophysiology. Annu Rev Nutr 32:229-243.

DOWNS, S.M., HUDSON, E.R. and HARDIE, D.G. (2002). A potential role for AMPactivated protein kinase in meiotic induction in mouse oocytes. Dev Biol 245: 200-212.

FEIGE, J.N., GELMAN, L., MICHALIK, L., DESVERGNE, B. and WAHLI, W. (2006) From molecular action to physiological outputs: peroxisome proliferator-activated receptors are nuclear receptors at the crossroads of key cellular functions. Prog Lipid Res 45: 120-159.

FROMENT, P., FABRE, S., DUPONT, J., PISSELET, C., CHESNEAU, D., STAELS, 
B. and MONGET, P. (2003). Expression and functional role of peroxisome proliferator-activated receptor-gamma in ovarian folliculogenesis in the sheep. Biol Reprod 69: 1665-1674.

GORALSKI, K.B., MCCARTHY, T.C., HANNIMAN, E.A., ZABEL, B.A., BUTCHER, E.C., PARLEE, S.D., MURUGANANDAN, S. and SINAL, C.J. (2007). Chemerin, a novel adipokine that regulates adipogenesis and adipocyte metabolism. $J$ Biol Chem 282: 28175-28188.

HARDIE, D.G. (2003). Minireview: the AMP-activated protein kinase cascade: the key sensor of cellular energy status. Endocrinology 144: 5179-5183.

KATSO, R., OKKENHAUG, K., AHMADI, K., WHITE, S., TIMMS, J. and WATERFIELD, M.D. (2001). Cellular function of phosphoinositide 3-kinases: implications for development, homeostasis, and cancer. Annu Rev Cell Dev Biol 17: 615-675.

KAYAMPILLY, P.P. and MENON, K.M. (2009). Follicle-stimulating hormone inhibits adenosine 5'-monophosphate-activated protein kinase activation and promotes cell proliferation of primary granulosa cells in culture through an Akt-dependent pathway. Endocrinology 150: 929-935.

KIM, E.K., PARK, J.M., LIM, S., CHOI, J.W., KIM, H.S., SEOK, H., SEO, J.K., OH, K., LEE, D.S., KIM, K.T. et al., (2011). Activation of AMP-activated protein kinase is essential for lysophosphatidic acid-induced cell migration in ovarian cancer cells. J Biol Chem 286: 24036-24045.

KIM, J., SATO, M., LI, Q., LYDON, J.P., DEMAYO, F.J., BAGCHI, I.C. and BAGCHI, M.K. (2008). Peroxisome proliferator-activated receptor gamma is a target of progesterone regulation in the preovulatory follicles and controls ovulation in mice. Mol Cell Biol 28: 1770-1782.

KOMAR, C.M. (2005). Peroxisome proliferator-activated receptors (PPARs) and ovarian function--implications for regulating steroidogenesis, differentiation, and tissue remodeling. Reprod Biol Endocrinol 3: 41.

MAILLARD, V., FROMENT, P., RAME, C., UZBEKOVA, S., ELIS, S. and DUPONT, J. (2011). Expression and effect of resistin on bovine and rat granulosa cell steroidogenesis and proliferation. Reproduction 141: 467-479.

MAILLARD, V., UZBEKOVA, S., GUIGNOT, F., PERREAU, C., RAME, C., COYRALCASTEL, S. and DUPONT, J. (2010). Effect of adiponectin on bovine granulosa cell steroidogenesis, oocyte maturation and embryo development. Reprod Biol Endocrinol 8: 23.

MAYES, M.A., LAFOREST, M.F., GUILLEMETTE, C., GILCHRIST, R.B. and RICHARD, F.J. (2007). Adenosine 5'-monophosphate kinase-activated protein kinase (PRKA) activators delay meiotic resumption in porcine oocytes. Biol Reprod 76: 589-597.

MOR, G., VISINTIN, I., LAI, Y., ZHAO, H., SCHWARTZ, P., RUTHERFORD, T., YUE, L., BRAY-WARD, P. and WARD, D.C. (2005). Serum protein markers for early detection of ovarian cancer. Proc Natl Acad Sci USA 102: 7677-7682.

MUNIR, I., YEN, H.W., BARUTH, T., TARKOWSKI, R., AZZIZ, R., MAGOFFIN, D.A. and JAKIMIUK, A.J. (2005). Resistin stimulation of 17alpha-hydroxylase activity in ovarian theca cells in vitro: relevance to polycystic ovary syndrome. J Clin Endocrinol Metab 90: 4852-4857.

OCÓN-GROVE, O.M., KRZYSIK-WALKER, S.M., MADDINENI, S.R., HENDRICKS, G.L. 3R.D., RAMACHANDRAN, R. (2008). Adiponectin and its receptors are expressed in the chicken testis: influence of sexual maturation on testicular ADIPOR1 and ADIPOR2 mRNA abundance. Reproduction. 136:627-638.

PIERRE, P., FROMENT, P., NEGRE, D., RAME, C., BARATEAU, V., CHABROLLE, C., LECOMTE, P. and DUPONT, J. (2009). Role of adiponectin receptors, AdipoR1 and AdipoR2, in the steroidogenesis of the human granulosa tumor cell line, KGN. Hum Reprod 24: 2890-2901.

REDDY, P., LIU, L., ADHIKARI, D., JAGARLAMUDI, K., RAJAREDDY, S., SHEN, Y., DU, C., TANG, W., HAMALAINEN, T., PENG, S.L. et al., (2008). Oocyte-specific deletion of Pten causes premature activation of the primordial follicle pool. Science 319: 611-613.

REVERCHON, M., CORNUAU, M., RAME, C., GUERIF, F., ROYERE, D. and DUPONT, J. (2012). Chemerin inhibits IGF-1-induced progesterone and estradiol secretion in human granulosa cells. Hum Reprod 27: 1790-1800.

ROMERO, I. and BAST, R.C., JR. (2012). Minireview: human ovarian cancer: biology, current management, and paths to personalizing therapy. Endocrinology 153: $1593-1602$.
RYAN, N.K., WOODHOUSE, C.M., VAN DER HOEK, K.H., GILCHRIST, R.B., ARMSTRONG, D.T. and NORMAN, R.J. (2002). Expression of leptin and its receptor in the murine ovary: possible role in the regulation of oocyte maturation. Biol Reprod 66: 1548-1554.

SCHERER, P.E., WILLIAMS, S., FOGLIANO, M., BALDINI, G. and LODISH, H.F. (1995). A novel serum protein similar to C1q, produced exclusively in adipocytes. J Biol Chem 270: 26746-26749.

SCHILFFARTH, S., ANTONI, B., SCHAMS, D., MEYER, H.H. and BERISHA, B. (2009). The expression of apelin and its receptor APJ during different physiological stages in the bovine ovary. Int J Biol Sci 5: 344-350.

SHACKELFORD, R.E., BUI, M.M., COPPOLA, D. and HAKAM, A. (2010). Overexpression of nicotinamide phosphoribosyltransferase in ovarian cancers. Int $J$ Clin Exp Pathol 3: 522-527.

SHEN, C.J., TSAI, E.M., LEE, J.N., CHEN, Y.L., LEE, C.H. and CHAN, T.F. (2010). The concentrations of visfatin in the follicular fluids of women undergoing controlled ovarian stimulation are correlated to the number of oocytes retrieved. Fertil Steril 93: 1844-1850.

SHIGETO, T., YOKOYAMA, Y., XIN, B. and MIZUNUMA, H. (2007). Peroxisome proliferator-activated receptor alpha and gamma ligands inhibit the growth of human ovarian cancer. Oncol Rep 18: 833-840.

SHIMIZU, T., KOSAKA, N., MURAYAMA, C., TETSUKA, M. and MIYAMOTO, A. (2009). Apelin and APJ receptor expression in granulosa and theca cells during different stages of follicular development in the bovine ovary: Involvement of apoptosis and hormonal regulation. Anim Reprod Sci 116: 28-37.

SPICER, L.J., SCHREIBER, N.B., LAGALY, D.V., AAD, P.Y., DOUTHIT, L.B. and GRADO-AHUIR, J.A. (2011). Effect of resistin on granulosa and theca cell function in cattle. Anim Reprod Sci 124: 19-27.

STEPPAN, C.M., BAILEY, S.T., BHAT, S., BROWN, E.J., BANERJEE, R.R., WRIGHT, C.M., PATEL, H.R., AHIMA, R.S. and LAZAR, M.A. (2001). The hormone resistin links obesity to diabetes. Nature 409: 307-312.

TABANDEH, M.R., HOSSEINI, A., SAEB, M., KAFI, M. and SAEB, S. (2010). Changes in the gene expression of adiponectin and adiponectin receptors (AdipoR1 and AdipoR2) in ovarian follicular cells of dairy cow at different stages of development. Theriogenology 73: 659-669.

TATEMOTO, K., HOSOYA, M., HABATA, Y., FUJII, R., KAKEGAWA, T., ZOU, M.X., KAWAMATA, Y., FUKUSUMI, S., HINUMA, S., KITADA, C. et al., (1998). Isolation and characterization of a novel endogenous peptide ligand for the human APJ receptor. Biochem Biophys Res Commun 251: 471-476.

TOSCA, L., CHABROLLE, C., UZBEKOVA, S. and DUPONT, J. (2007a). Effects of metformin on bovine granulosa cells steroidogenesis: possible involvement of adenosine 5' monophosphate-activated protein kinase (AMPK). Biol Reprod 76: 368-378.

TOSCA, L. UZBEKOVA, S., CHABROLLE, C., DUPONT, J. (2007b). Possible role of 5'AMP-activated protein kinase in the metformin-mediated arrest of bovine oocytes at the germinal vesicle stage during in vitro maturation. Biol Reprod 77: 452-465.

TOSCA, L., FROMENT, P., SOLNAIS, P., FERRE, P., FOUFELLE, F. and DUPONT, J. (2005). Adenosine 5'-monophosphate-activated protein kinase regulates progesterone secretion in rat granulosa cells. Endocrinology 146: 4500-4513.

TOSCA, L., RAME, C., CHABROLLE, C., TESSERAUD, S. and DUPONT, J. (2010). Metformin decreases IGF1-induced cell proliferation and protein synthesis through AMP-activated protein kinase in cultured bovine granulosa cells. Reproduction 139: 409-418

YABA, A., BIANCHI, V., BORINI, A. and JOHNSON, J. (2008). A putative mitotic checkpoint dependent on mTOR function controls cell proliferation and survival in ovarian granulosa cells. Reprod Sci 15: 128-138.

ZHANG, G.Y., AHMED, N., RILEY, C., OLIVA, K., BARKER, G., QUINN, M.A. and RICE, G.E. (2005). Enhanced expression of peroxisome proliferator-activated receptor gamma in epithelial ovarian carcinoma. Br J Cancer 92: 113-119.

ZHENG, W., NAGARAJU, G., LIU, Z. and LIU, K. (2012). Functional roles of the phosphatidylinositol 3-kinases (PI3Ks) signaling in the mammalian ovary. Mol Cell Endocrinol 356: 24-30. 


\section{Further Related Reading, published previously in the Int. J. Dev. Biol.}

Impaired meiotic competence in putative primordial germ cells produced from mouse embryonic stem cells Marianna Tedesco, Donatella Farini and Massimo De Felici Int. J. Dev. Biol. (2011) 55: 215-222

Differentiation of mouse primordial germ cells into female or male germ cells N Nakatsuji and S Chuma

Int. J. Dev. Biol. (2001) 45: 541-548

The meiotic specific synaptonemal complex protein SCP3 is expressed by female and male primordial germ cells of the mouse embryo

A D Di Carlo, G Travia and M De Felici

Int. J. Dev. Biol. (2000) 44: 241-244

In vitro germ cell differentiation during sex differentiation in a teleost fish Tohru Kobayashi

Int. J. Dev. Biol. (2010) 54: 105-111

Generation of germ-line chimera zebrafish using primordial germ cells isolated from cultured blastomeres and cryopreserved embryoids

Yutaka Kawakami, Rie Goto-Kazeto, Taiju Saito, Takafumi Fujimoto, Shogo Higaki, Yoshiyuki Takahashi, Katsutoshi Arai and Etsuro Yamaha

Int. J. Dev. Biol. (2010) 54: 1493-1501

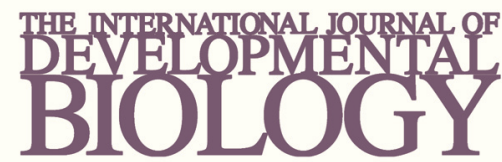

Volume 54 Nos. $6 / 7$
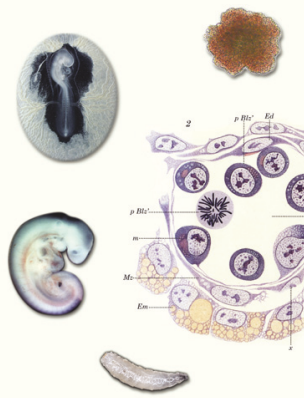

(2) (8)
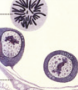

(1)
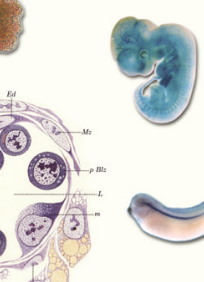

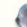
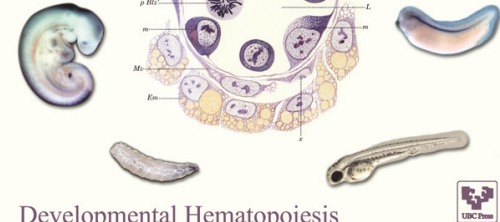

Developmental Hematopoiesis
5 yr ISI Impact Factor $(2011)=2.959$

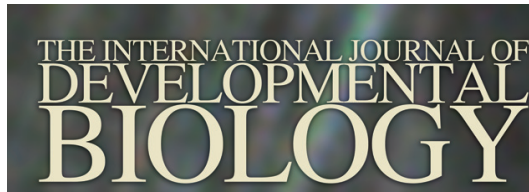

Special Issue

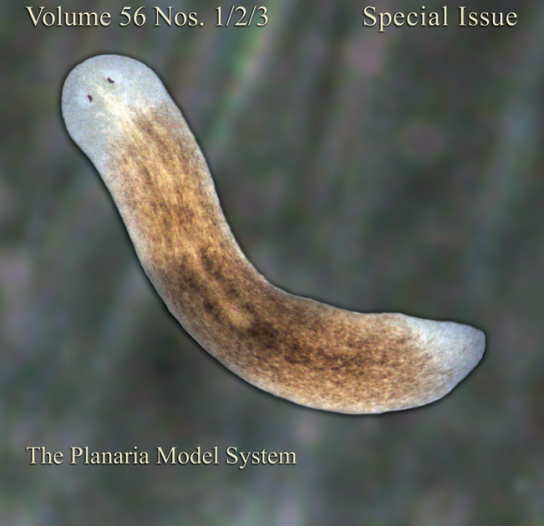

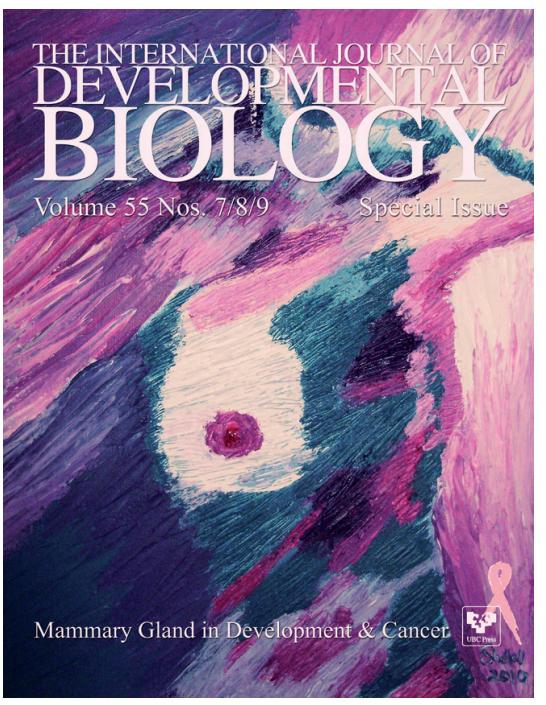

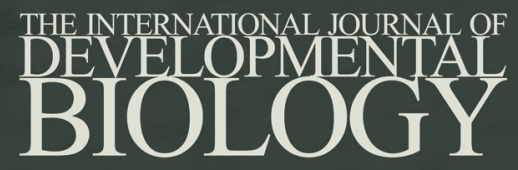

Volume 55 Nos. $4 / 5$

Special Issue

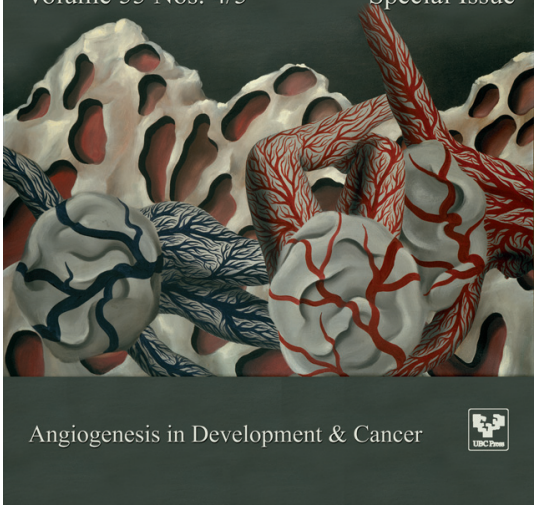

